\title{
Overestimates for the Gain of Multiple Linear Approximations
}

S. Murphy

Technical Report

RHUL-MA-2009-21

16 October 2009 


\section{Abstract}

We show that Corollary 1 of "On Multiple Linear Approximations" (Crypto 2004 - LNCS 3152) is incorrect. In particular, the value given for the gain by Corollary 1 is likely to be a significant overestimate of this quantity. Thus any data requirements for linear cryptanalysis with multiple linear approximations based on this value for the gain are highly questionable.

\section{Introduction}

Linear cryptanalysis [4] of a block cipher in its basic form uses a linear approximation of the form

$$
\alpha^{T}\left(\begin{array}{l}
\mathbf{p} \\
\mathbf{c}
\end{array}\right)=k \text { with probability } \frac{1}{2}(1+\epsilon),
$$

where $\alpha$ is a data mask, $k$ is one bit of key information, $\mathbf{p}$ is a plaintext and $\mathbf{c}$ is a corresponding ciphertext. The value $\epsilon$ is known as the imbalance or correlation (twice the bias) of the linear approximation. If $\epsilon \neq 0$, then it is possible to estimate the key bit $k$ reasonably accurately if the number $N$ of plaintext-ciphertext pairs is at least $\epsilon^{-2}[4]$.

Enhanced forms of linear cryptanalysis [2,3] use a collection of $m$ such linear approximations. Such a situation with multiple linear approximations is also considered by [1], where the gain of such a linear cryptanalysis is defined. The gain is a attempt to quantify the advantage of a such a linear cryptanalysis over exhaustive search.

This paper is concerned with the values given for the gain by [1]. In particular, we show that the value for the gain given there by Corollary 1 generally greatly exceeds the value for the gain given there by Theorem 1 .

\section{Multiple Linear Approximations}

We consider a linear cryptanalysis based on $N$ plaintext-ciphertext pairs. We suppose that we have $m$ linear approximations

$$
\alpha_{i}^{T}\left(\begin{array}{l}
\mathbf{p} \\
\mathbf{c}
\end{array}\right)=k_{i} \text { with probability } \frac{1}{2}\left(1+\epsilon_{i}\right)
$$

for distinct data masks $\alpha_{i}$, individual bits of key information $k_{i}$ and imbalances $\epsilon_{i}(i=1, \ldots, m)$. The capacity $\bar{c}^{2}$ of this collection of linear approximations is given by Definition 2 of [1] to be $\bar{c}^{2}=\sum_{i=1}^{m} \epsilon_{i}^{2}$.

For simplicity, we suppose that the $m$ key bits $k_{1}, \ldots, k_{m}$ give $m$ bits of information about the block cipher key. We let $\mathbf{z}=\left(k_{1}, \ldots, k_{m}\right)^{T}$ denote the key class, and we denote the set of all key classes by $\mathcal{Z}$, so $\mathcal{Z}=\mathbb{Z}_{2}^{m}$ and $|\mathcal{Z}|=2^{m}$. We let $\mathbf{z}^{*}$ denote the key class containing the true key, and, without loss of generality, we suppose that $\mathbf{z}^{*}=0$. We let $\mathcal{Z}^{*}=\mathcal{Z} \backslash\left\{\mathbf{z}^{*}\right\}=\mathcal{Z}_{2}^{m} \backslash\{0\}$ denote 
the set of key classes not containing the true key, so $\left|\mathcal{Z}^{*}\right|=2^{m}-1$. We denote the $m$-dimensional imbalance vector corresponding to key class $\mathbf{z}$ by $\mathbf{c}_{\mathbf{z}}$, so

$$
\mathbf{c}_{z}=\left((-1)^{z_{1}} \epsilon_{1}, \ldots,(-1)^{z_{m}} \epsilon_{m}\right)^{T} .
$$

We note that the squared distance from such an imbalance vector to the imbalance vector for the true key class is given by

$$
\left|\mathbf{c}_{\mathbf{z}}-\mathbf{c}_{\mathbf{z}^{*}}\right|^{2}=\left|\mathbf{c}_{\mathbf{z}}-\mathbf{c}_{0}\right|^{2}=\left|-2\left(z_{1} \epsilon_{1}, \ldots, z_{m} \epsilon_{m}\right)^{T}\right|^{2}=4 \sum_{i=1}^{m} z_{i}^{2} \epsilon_{i}^{2} .
$$

\section{Mathematical Concepts used to Define Gain Values}

The values given for the gain by [1] can be expressed in terms of two functions, $g$ and $H_{m}$, and a random variable $X$, which we now define.

The function $g$ on the positive real numbers is defined by

$$
g(x)=\phi\left(-\frac{1}{2} N^{\frac{1}{2}} x^{\frac{1}{2}}\right),
$$

where $\phi$ denotes the cumulative distribution function for a standard normal $\mathrm{N}(0 ; 1)$ random variable. We note that $g(x)$ is a convex function of $x$ for $x>0$ as

$$
g^{\prime \prime}(x)=\frac{1}{32} \frac{1}{\sqrt{2 \pi}} N^{\frac{1}{2}} e^{-\frac{N x}{8}}\left(N+4 x^{-1}\right) x^{-\frac{1}{2}}>0 \text { for } x>0 .
$$

The function $H_{m}$ on the positive real numbers is defined by

$$
H_{m}(x)=-\log _{2}\left(2\left(1-2^{-m}\right) x+2^{-m}\right)=-\log _{2}\left[2 \frac{\left|Z^{*}\right|}{|Z|} x+\frac{1}{|Z|}\right] .
$$

We note that $H_{m}(x)$ is a decreasing function of $x$ for $x>0$ as

$$
H_{m}^{\prime}(x)=-\frac{1}{\log 2}\left(\frac{2\left(1-2^{-m}\right)}{2\left(1-2^{-m}\right) x+2^{-m}}\right)<0 \text { for } x>0 .
$$

The random variable $X$ is defined by

$$
X=\left|\mathbf{c}_{\mathbf{z}}-\mathbf{c}_{\mathbf{z}^{*}}\right|^{2} \text { with probability }\left|Z^{*}\right|^{-1}=\left(2^{m}-1\right)^{-1} \text { for } \mathbf{z} \in \mathcal{Z}^{*} .
$$

Thus $X$ is the random variable giving the squared distance of an imbalance vector for an incorrect key class from the imbalance vector for the true key class.

\section{Comparison of Values for the Gain}

We now compare the two values given for the gain in Theorem 1 and Corollary 1 of [1]. We show in Appendix A that the value $\gamma$ for the gain given by Theorem 1 is given by

$$
\gamma=H_{m}(\mathbf{E}[g(X)]) .
$$


We show in Appendix B that the value $\widetilde{\gamma}$ for the gain given by Corollary 1 is given by

$$
\widetilde{\gamma}=H_{m}\left(g\left(2 \bar{c}^{2}\right)\right)=H_{m}\left(g\left(\left(1-2^{-m}\right) \mathbf{E}[X]\right)\right) .
$$

However, this value $\widetilde{\gamma}$ for the gain can be well approximated by $\widehat{\gamma}$, where

$$
\widehat{\gamma}=H_{m}(g(\mathbf{E}[X])) .
$$

We now use Jensen's inequality [6] to compare $\gamma$ and $\widehat{\gamma}$. As $g$ is a convex function of the positive real numbers, Jensen's inequality shows that

$$
g(\mathbf{E}[X]) \leq \mathbf{E}[g(X)] .
$$

Furthermore $H_{m}$ is a decreasing function of the positive real numbers, so

$$
\widehat{\gamma}=H_{m}(g(\mathbf{E}[X])) \geq H_{m}(\mathbf{E}[g(X)])=\gamma .
$$

However, $\widetilde{\gamma}$ is usually extremely well-approximated by $\widehat{\gamma}$, so giving Lemma 1 .

Lemma 1. The value $\widetilde{\gamma}$ for the gain given by Corollary 1 generally exceeds the value $\gamma$ given for the gain by Theorem 1 .

\section{Example Values for the Gain}

The important issue in the use of Corollary 1 of [1] to give the gain is whether the overestimate of $\gamma$ by $\widetilde{\gamma}$ referred to in Lemma 1 gives a significant error in the value of the gain. We show by giving an example that it is indeed generally the case that the use of $\widetilde{\gamma}$ given in Corollary 1 gives a large overestimate of the gain $\gamma$ given by Theorem 1 .

For simplicity, we assume that all $m$ linear approximations have the same imbalance $\epsilon$, that is $\epsilon_{1}=\ldots=\epsilon_{m}=\epsilon$. The capacity of such a collection of linear approximations is clearly $\bar{c}^{2}=m \epsilon^{2}$. In this situation, using the result given in Section 2, we have

$$
\left|\mathbf{c}_{\mathbf{z}}-\mathbf{c}_{\mathbf{z}^{*}}\right|^{2}=4 \epsilon^{2} \sum_{i=1}^{m} z_{i}^{2}=4 \epsilon^{2}|\mathbf{z}|^{2} .
$$

As there are $\left(\begin{array}{c}m \\ l\end{array}\right)$ such vectors $\mathbf{z} \in \mathbb{Z}_{2}^{m}$ with $|\mathbf{z}|^{2}=l$, the random variable $X$ is given by

$$
X=4 \epsilon^{2} l \text { with probability }\left(\begin{array}{c}
m \\
l
\end{array}\right)\left(2^{m}-1\right)^{-1} \quad[l=1, \ldots, m] .
$$

Thus $X$ is a multiple of a censored $\operatorname{Bin}\left(m, \frac{1}{2}\right)$ random variable with 0 removed, so the mean of $X$ is given by $\mathbf{E}[X]=4 \epsilon^{2} \frac{m}{2} \frac{2^{m}}{2^{m}-1}$. We therefore obtain $g(\mathbf{E}[X])$, used to define $\widehat{\gamma}$, as

$$
\begin{aligned}
g(\mathbf{E}[X]) & =\phi\left(-\frac{1}{2} N^{\frac{1}{2}} \epsilon\left(2 m \frac{2^{m}}{2^{m}-1}\right)^{\frac{1}{2}}\right) \\
& =\phi\left(-\left(N \epsilon^{2}\right)^{\frac{1}{2}}\left(\frac{m}{2}\right)^{\frac{1}{2}}\left(\frac{2^{m}}{2^{m}-1}\right)^{\frac{1}{2}}\right) .
\end{aligned}
$$


By contrast, the mean of $g(X)$ is given by

$$
\begin{aligned}
\mathbf{E}[g(X)] & =\frac{1}{2^{m}-1} \sum_{l=1}^{m}\left(\begin{array}{c}
m \\
l
\end{array}\right) \phi\left(-\frac{1}{2} N^{\frac{1}{2}} 2 \epsilon l^{\frac{1}{2}}\right) \\
& =\frac{1}{2^{m}-1} \sum_{l=1}^{m}\left(\begin{array}{c}
m \\
l
\end{array}\right) \phi\left(-\left(N \epsilon^{2}\right)^{\frac{1}{2}} l^{\frac{1}{2}}\right) .
\end{aligned}
$$

We now consider the values of these two expressions for a particular example. We suppose that there are $m=8$ linear approximations, so the capacity $\bar{c}^{2}=$ $8 \epsilon^{2}$. We further suppose that we have $N=2 \epsilon^{-2}$ plaintext-ciphertext pairs, so $N \epsilon^{2}=2$. In this case we have

$$
g(\mathbf{E}[X])=0.0023 \text {, whereas } \mathbf{E}[g(X)]=0.0074 .
$$

For this example, we have $g(\mathbf{E}[X])>3 \mathbf{E}[g(X)]$, so illustrating Jensen's inequality. However, despite Jensen's inequality, it is essentially asserted by the "proof" of Corollary 1 that $g(\mathbf{E}[X])=\mathbf{E}[g(X)]$. (We note that the function $f(x)=g(-x)$ is erroneously used in this "proof" instead of $g(x)$.) This example shows that this assertion, which is the basis of the "proof" of Corollary 1, is simply wrong. As with the discussion by [1] of probabilities for dependent data masks [5], the given "proof" of Corollary 1 by [1] is not correct.

We now calculate the various values given for the gain in this situation, so

$$
\gamma=H_{8}(g(\mathbf{E}[X]))=5.75 \text { and } \widehat{\gamma}=H_{8}=(\mathbf{E}[g(X)])=6.88 .
$$

Furthermore, a direct calculation gives $\widetilde{\gamma}=6.87$, so $\widehat{\gamma}$ is obviously a very good approximation of $\widetilde{\gamma}$. In this situation, Corollary 1 overestimates the gain as given by Theorem 1 by over one bit in six.

\section{Conclusions}

We have shown that the value for the gain given by Corollary 1 of [1] is not reliable, and is in general a large overestimate of the value of the gain given by Theorem 1. Furthermore, the "proof" given of Corollary 1 simply ignores Jensen's inequality, a fundamental result in probability and theoretical statistics. Any result based on this value for the gain given by Corollary 1, such as the theoretical data requirements for such a linear crypanalysis, is therefore highly questionable.

\section{References}

1. A. Biryukov, C. De Cannière, and M. Quiquater. On Multiple Approximations. In M. Franklin, editor, Advances in Cryptology - CRYPTO 04, volume 3152 of LNCS, pages 1-22. Springer-Verlag, 2004.

2. B.S. Kaliski and M.J.B. Robshaw. Linear Cryptanalysis Using Multiple Approximations. In Y. Desmedt, editor, Advances in Cryptology - CRYPTO 94, volume 839 of $L N C S$, pages 26-39. Springer-Verlag, 1994. 
3. B.S. Kaliski and M.J.B. Robshaw. Linear Cryptanalysis Using Multiple Approximations and FEAL. In B. Preneel, editor, Fast Software Encryption 1994, volume 1008 of LNCS, pages 249-264. Springer-Verlag, 1995.

4. M. Matsui. Linear Cryptanalysis for the DES Cipher. In T. Helleseth, editor, Advances in Cryptology - EUROCRYPT 1993, volume 765 of LNCS, pages 386397. Springer-Verlag, 1993.

5. S. Murphy. The Independence of Linear Approximations in Symmetric Cryptology. IEEE Transactions on Information Theory, 52:5510-5518, 2006.

6. S.D. Silvey. Statistical Inference. Chapman and Hall, 1975.

\section{A Value for the Gain given by Theorem 1}

The value $\gamma$ given for the gain given by Theorem 1 of [1] is

$$
\gamma=-\log _{2}\left[2 \frac{1}{|\mathcal{Z}|} \sum_{\mathbf{z} \in \mathcal{Z}^{*}} \phi\left(-\frac{1}{2} N^{\frac{1}{2}}\left|\mathbf{c}_{\mathbf{z}}-\mathbf{c}_{\mathbf{z}^{*}}\right|\right)+\frac{1}{|\mathcal{Z}|}\right]
$$

Thus we have

$$
\gamma=-\log _{2}\left[2\left(1-2^{-m}\right) \frac{1}{2^{m}-1} \sum_{\mathbf{z} \neq 0} g\left(\left|\mathbf{c}_{\mathbf{z}}-\mathbf{c}_{0}\right|^{2}\right)+2^{-m}\right] .
$$

However, the mean value of $g(X)$ is given by

$$
\mathbf{E}[g(X)]=\frac{1}{2^{m}-1} \sum_{\mathbf{z} \neq 0} g\left(\left|\mathbf{c}_{\mathbf{z}}-\mathbf{c}_{0}\right|^{2}\right),
$$

so we have shown that the value $\gamma$ given for the gain by Theorem 1 is given by

$$
\gamma=-\log _{2}\left[2\left(1-2^{-m}\right) \mathbf{E}[g(X)]+2^{-m}\right]=H_{m}(\mathbf{E}[g(X)]) .
$$

\section{B Value for the Gain given by Corollary 1}

The value $\widetilde{\gamma}$ given for the gain by Corollary 1 of [1] is

$$
\begin{aligned}
\widetilde{\gamma} & =-\log _{2}\left[2 \frac{|\mathcal{Z}|-1}{|\mathcal{Z}|} \phi\left(-\left(\frac{1}{2} N \bar{c}^{2}\right)^{\frac{1}{2}}\right)+\frac{1}{|\mathcal{Z}|}\right] \\
& =H_{m}\left(\phi\left(-\frac{1}{2}\left(2 N \bar{c}^{2}\right)^{\frac{1}{2}}\right)\right)=H_{m}\left(g\left(2 \bar{c}^{2}\right)\right) .
\end{aligned}
$$

We express this quantity in terms of the mean of $X$, which is given by

$$
\mathbf{E}[X]=\frac{1}{\left|\mathcal{Z}^{*}\right|} \sum_{\mathbf{z} \neq \mathbf{z}^{*}}\left|\mathbf{c}_{\mathbf{z}}-\mathbf{c}_{\mathbf{z}^{*}}\right|^{2}=\left(2^{m}-1\right)^{-1} \sum_{\mathbf{z} \neq 0}\left|\mathbf{c}_{\mathbf{z}}-\mathbf{c}_{0}\right|^{2} .
$$

However, $\left|\mathbf{c}_{\mathbf{z}}-\mathbf{c}_{0}\right|^{2}=4 \sum_{i=1}^{m} z_{i}^{2} \epsilon_{i}^{2}$ (Section 1), so we have

$$
\mathbf{E}[X]=\left(2^{m}-1\right)^{-1} \sum_{\mathbf{z} \neq 0} \sum_{i=1}^{m} 4 z_{i}^{2} \epsilon_{i}^{2}=\frac{4}{2^{m}-1} \sum_{i=1}^{m} \epsilon_{i}^{2} \sum_{\mathbf{z} \neq 0} z_{i}^{2}=\frac{4 \cdot 2^{m-1}}{2^{m}-1} \bar{c}^{2}
$$


as the two summations in the above expression can be evaluated to give

$$
\sum_{\mathbf{z} \neq 0} z_{i}^{2}=\sum_{\mathbf{z} \neq 0} z_{i}=2^{m-1} \text { and } \sum_{i=1}^{m} \epsilon_{i}^{2}=\bar{c}^{2} .
$$

Thus we can give the capacity in terms of the mean of $X$ as

$$
\bar{c}^{2}=\frac{1}{2}\left(1-2^{-m}\right) \mathbf{E}[X]
$$

so we can obtain

$$
\phi\left(-\left(\frac{1}{2} N \bar{c}^{2}\right)^{\frac{1}{2}}\right)=\phi\left(-\frac{1}{2} N^{\frac{1}{2}}\left(\left(1-2^{-m}\right) \mathbf{E}[X]\right)^{\frac{1}{2}}\right)=g\left(\left(1-2^{-m}\right) \mathbf{E}[X]\right) .
$$

This means we can express the value $\widetilde{\gamma}$ given for the gain by Corollary 1 as

$$
\widetilde{\gamma}=H_{m}\left(g\left(\left(1-2^{-m}\right) \mathbf{E}[X]\right)\right) .
$$

If we now define the value

$$
\widehat{\gamma}=H_{m}(g(\mathbf{E}[X])),
$$

then clearly $\widehat{\gamma}$ is a very good approximation of $\widetilde{\gamma}$ when $m$ is moderately large. 